Research Paper

\title{
How Much Colonic Redundancy Could Be Obtained by Splenic Flexure Mobilization in Laparoscopic Anterior or Low Anterior Resection?
}

\author{
Bong-Hyeon Kye ${ }^{1}$, Hyung-Jin Kim¹, Hyun-Sil Kim${ }^{1}$, Jun-Gi Kim², Hyeon-Min Cho ${ }^{1 凶}$ \\ 1. St. Vincent Hospital, Department of Surgery, College of Medicine, The Catholic University of Korea, Suwon, Korea \\ 2. Seoul St. Mary's Hospital, Department of Surgery, College of Medicine, The Catholic University of Korea, Seoul, Korea
}

$\square$ Corresponding author: Hyeon-Min Cho, MD, Department of Surgery, St. Vincent's Hospital, The Catholic University of Korea, Suwon, Korea, 93-6 Ji-dong, Paldal-gu, Suwon-si, Gyeonggi-do, Korea. Tel: 82-31-249-7170; Fax: 82-31-247-5347; E-mail: hmcho@catholic.ac.kr

( ) Ivyspring International Publisher. This is an open-access article distributed under the terms of the Creative Commons License (http://creativecommons.org/ licenses/by-nc-nd/3.0/). Reproduction is permitted for personal, noncommercial use, provided that the article is in whole, unmodified, and properly cited.

Received: 2014.02.18; Accepted: 2014.05.12; Published: 2014.06.09

\begin{abstract}
Background and Objectives: Splenic flexure mobilization (SFM) is performed to ensure a tension free anastomosis with an adequate resection margin in laparoscopic anterior resection (AR) or low anterior resection (LAR). This retrospective study was performed to determine the amount of colonic redundancy that can be expected by SFM.

Methods: Retrospective review of medical record for a total of 203 patients who underwent SFM during laparoscopic AR or LAR for the treatment of sigmoid colon or rectal cancer was performed.

Results: The obtained redundancy of the colon by SFM was $27.81 \pm 7.29 \mathrm{~cm}$ from the sacral promontory. The redundancy of the colon by SFM with high ligation of the inferior mesenteric vein (IMV) (29.54 $\pm 7.17 \mathrm{~cm}$ from the sacral promontory) was greater than that with low ligation of the IMV (24.94 $\pm 6.07 \mathrm{~cm}$ from the sacral promontory, $\mathrm{P}<0.000 \mathrm{I})$. It took about $9.82 \%$ of the total operation time to perform SFM. There was no intraoperative complication during SFM.

Conclusions: SFM during laparoscopic AR or LAR is a safe and feasible option. Based on the result of this study, one can gain about $27.8 \mathrm{I} \mathrm{cm}$ redundancy of the colon by SFM.
\end{abstract}

Key words: laparoscopic anterior resection, laparoscopic low anterior resection, splenic flexure mobilization

\section{Introduction}

Securing adequate mobilization and preserving the blood supply to the organ ends are required to ensure safe gastrointestinal anastomosis [1]. Colorectal anastomosis failure contributes to both the morbidity and mortality of patients undergoing rectal surgery and the increased risk of local cancer recurrence [2]. The use of splenic flexure mobilization (SFM) during anterior resection (AR) or low anterior resection (LAR) can facilitate tension-free anastomosis with an adequate cancer-free margin by straightening a splenic flexure colon. SFM was routinely performed in the past; however, some surgeons now use it only when necessary because it is a difficult step within both conventional and laparoscopic procedures and may require more time, patient repositioning, a longer incision, or additional port insertion [3, 4]. SFM is considered more difficult in laparoscopic colorectal resection than in open surgery and is usually done only in selective cases [5]. According to a mail-in survey completed by 35 experienced laparoscopic colorectal surgeons, SFM is one of the hardest procedures to perform [6]. However, many surgeons still 
believe that SFM is necessary to ensure a tension-free and well-vascularized anastomosis. Considering this controversy, we believe it necessary to determine how much colonic redundancy can be obtained by SFM to identify whether colorectal surgeons should do it or not.

Two cadaveric studies recently demonstrated the degree to which the use of SFM could lengthen the colon $[7,8]$. However, no study has measured colon elongation by SFM during actual surgery, especially in laparoscopic surgery. In this study, we aimed to determine the possible degree of obtainable redundancy of the colon by SFM in laparoscopic AR or LAR and evaluate the safety of SFM.

\section{Patients and Methods}

\section{Patient enrollment}

The data of 203 patients concerning colon elongation by SFM in laparoscopic AR or LAR at the Department of Surgery, St. Vincent's Hospital, The Catholic University of Korea, from July 2009 to November 2012 were available. 110 of these patients had rectal cancer, 32 had rectosigmoid junction colon cancer, and 61 had sigmoid colon cancer. Of the 110 patients with rectal cancer, 70 underwent neoadjuvant chemoradiotherapy. After receiving institutional review board approval, we studied retrospectively these 203 patients who had undergone laparoscopic AR or LAR with SFM for the treatment of sigmoid colon or rectal cancer.

\section{Surgical procedure}

All the surgeries were performed by 3 colorectal surgeons.

\section{I) Mobilization of the mesocolon}

Before dissecting the colon, we placed a surgical clip on a sigmoid-descending (SD) colon junction to mark the spot "A" (Figure 1). After performing high ligation of the inferior mesenteric artery (IMA), we separated the retroperitoneum from the mesocolon of the descending and transverse colon from the medial to the lateral side. We thought it best to perform this procedure after the high ligation since the avascular plane can be found more easily. We dissected the retroperitoneum laterally to the level of the descending colon and then to the lower border of the pancreas superiorly. Next we detached the descending colon from the parietal peritoneum. Since the retroperitoneal dissection had been performed from the medial side, this was completed without much difficulty.

\section{2) Mobilization of the splenic flexure}

After detaching the colon from the parietal peritoneum, we changed the patient's position into a reverse Trendelenburg position, detached the omentum from the transverse colon starting at the level of the falciform ligament and extending to the splenic flexure colon, and then entered the lesser sac. We subsequently separated the lower border of the pancreas and the transverse mesocolon to the point at which the inferior mesenteric vein (IMV) and splenic vein were visible.

\section{3) Ligation of the inferior mesenteric vein}

After moving the patient back to the Trendelenburg position, we ligated the IMV at the level below the pancreas. We then resected the mesocolon up to the marginal vessel. Here we regarded ligation of the IMV after the left colic vein converged to the IMV as a high ligation and ligation of the IMV before the left colic vein converged to the IMV as a low ligation. When we performed high ligation of the IMV, we always ligated the left colic vein and artery in the same operational field as the IMV ligation (Fig. 2).

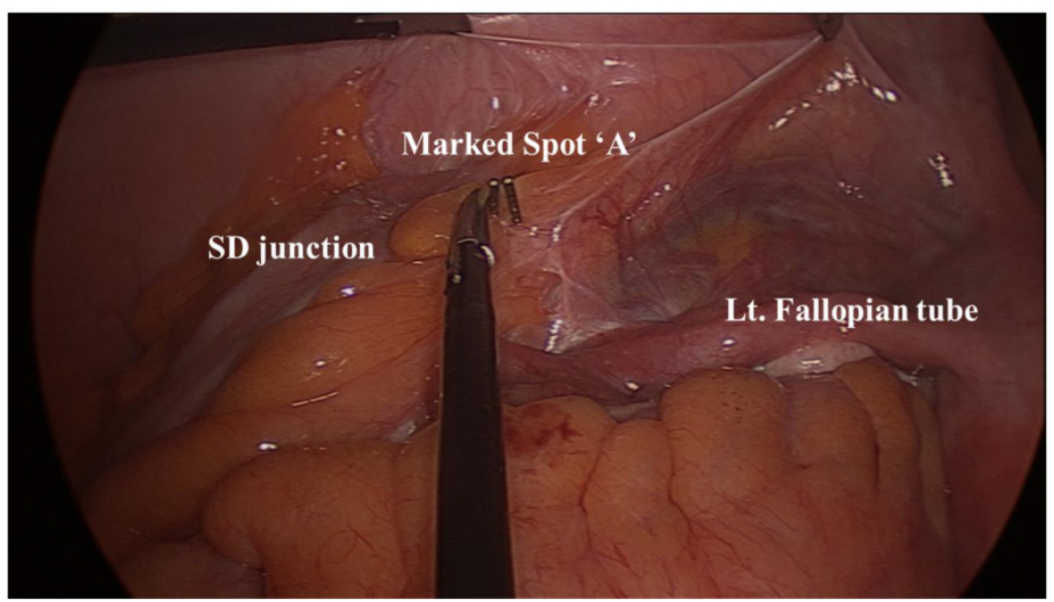

Figure I. Before colon mobilization, we placed a surgical clip on the sigmoid-descending colon junction to mark the spot "A." 


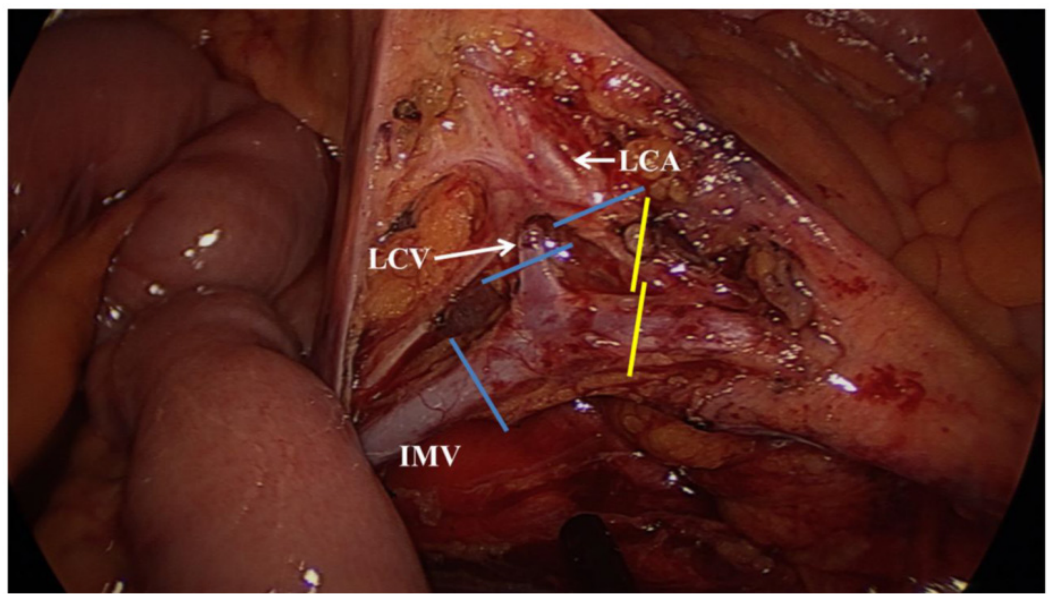

Figure 2. We regarded the ligation of the inferior mesenteric vein (IMV) after the left colic vein (LCV) was converged to the IMV as a high ligation. When we performed a high IMV ligation, we always ligated the LCV and left colic artery (LCA) in the same operative field of the IMV ligation (blue lines). Ligation of the IMV before the LCV was converged to IMV was regarded as a low ligation. In low ligation of IMV, we ligated LCA, but did not ligate LCV (yellow lines).
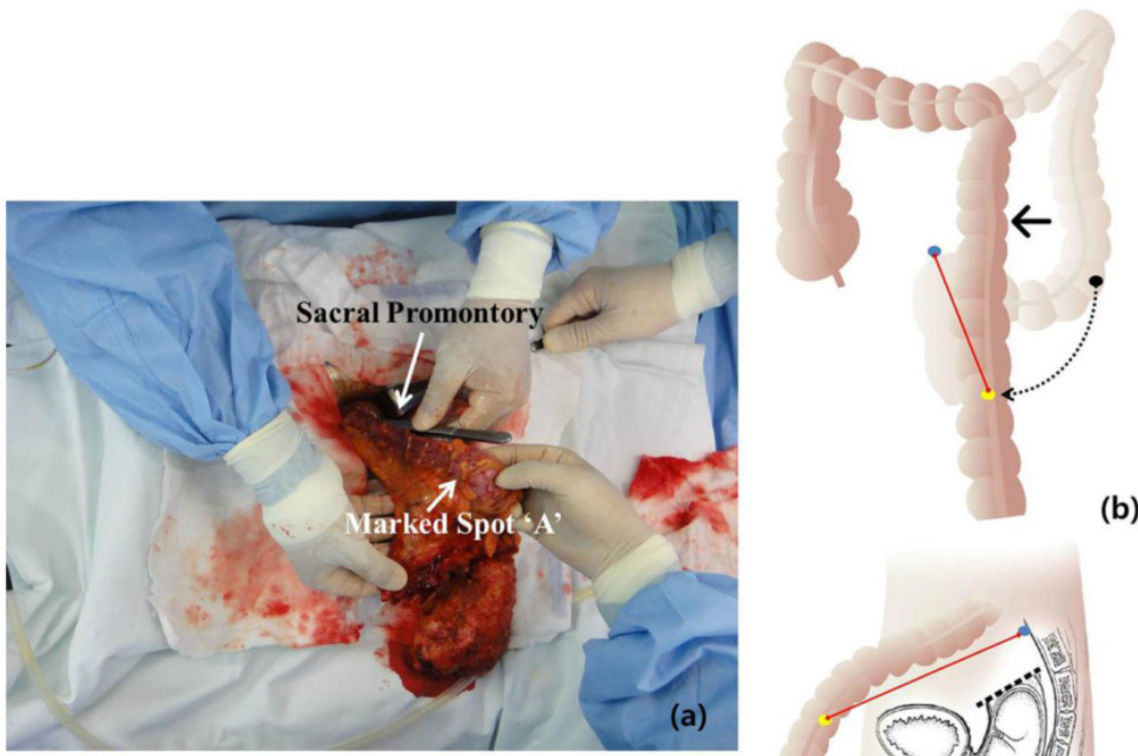

(b)

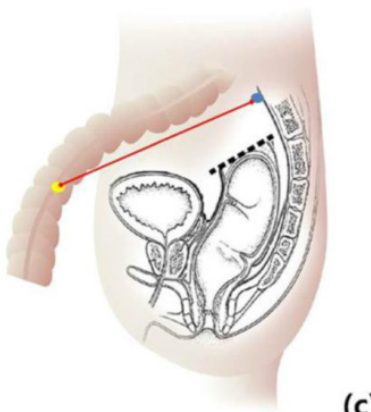

(c)

Figure 3. The specimen was delivered to the extraperitoneal space through a Pfannenstiel incision. To determine the obtained redundancy of the colon after splenic flexure mobilization, the measurement was taken from the sacral promontory to the marked spot " $A$ " by using 25 -cm long forceps (a). The mobilized colon after splenic flexure mobilization is shown schematically in (b) and (c). The black dot means marked spot " $A$ " before splenic flexure mobilization as initial sigmoid-descending junction and the yellow dot means marked spot " $A$ " after splenic flexure mobilization. The blue dot means sacral promontory. The obtained redundancy of the colon is measured as the distance between the yellow dot and the blue dot (red line).

\section{4) Measurement of the length of the elongated colon}

After we resected the rectum by using laparoscopic stapling devices, the specimen was delivered to the extraperitoneal space through a Pfannenstiel incision. Measurement of the obtained redundancy of the colon after SFM was done from the sacral promontory to the marked spot "A" (Fig. 3).

\section{5) Other}

After measuring the obtained redundancy of the colon, we resected the proximal colon, retained an adequate resection margin, and performed anastomosis by using a DST EEA ${ }^{\mathrm{TM}}$ (Covidien, Mansfield, USA). When a patient underwent neoadjuvant chemoradiotherapy before the surgery or the anastomosis was $<5 \mathrm{~cm}$ from the anal verge, we created a temporary diverting stoma.

\section{Parameters}

We analyzed the following data: 1) distance between the marked spot " $\mathrm{A}$ " and the sacral promontory after SFM; 2) distal resection margin; 3) proximal 
resection margin; 4) the length of specimen; 5) total operation time; 6) the time required for SFM; and 7) the ratio of the time required for SFM and total operation time. Distal resection margin, proximal resection margin, and the length of specimen were measured immediately after the resection (before fixation for pathologic evaluation). We analyzed the intra- and postoperative complications, especially anastomosis leakage.

\section{Statistical analysis}

Continuous variables were compared by using Student's $t$-test and expressed as means \pm SD. Categorical variables were analyzed by using the $\chi^{2}$ test. Significance was defined as values of $\mathrm{P} \leq 0.05$. All statistical analyses were performed by using the Statistical Package of the Social Sciences (SPSS) version 12.0 for Windows (SPSS, Inc., Chicago, IL, USA).

\section{Results}

This study included 121 males and 82 females. The mean patient age was $64.56 \pm 11.11$ years (range, $36-90)$. The mean body mass index was $24.03 \pm 3.62$ $\mathrm{kg} / \mathrm{m} 2$. Table 1 shows the data of all 203 patients enrolled in this study. The obtained redundancy of the colon by SFM was $27.81 \pm 7.29 \mathrm{~cm}$. The mean time required for SFM was approximately $10 \%$ of the entire operation time.

Table I. Data of 203 patients who underwent laparoscopic splenic flexure mobilization

\begin{tabular}{|c|c|c|}
\hline & Mean \pm S.D. & Range \\
\hline $\mathrm{BMI}^{\dagger}$ & $24.03 \pm 3.62$ & $14.5-35.5$ \\
\hline From SP $(\mathrm{cm})$ & $27.81 \pm 7.29$ & $9-59$ \\
\hline DRMs $(\mathrm{cm})$ & $6.83 \pm 4.45$ & $0.4-35.5$ \\
\hline PRM" (cm) & $16.18 \pm 6.83$ & $2-40$ \\
\hline Specimen length (cm) & $25.90 \pm 5.57$ & $10-45$ \\
\hline Operation time (minutes) & $236.86 \pm 54.31$ & $145-475$ \\
\hline The time required for SFMף (minutes) & $25.61 \pm 10.56$ & $9-60$ \\
\hline The time required for SFM / Operation time (\%) & $9.82 \pm 3.94$ & $2.9-27.9$ \\
\hline
\end{tabular}

The obtained redundancy of the colon in the high ligation group $(29.54 \pm 7.17 \mathrm{~cm})$ was more than that in low ligation group $(24.94 \pm 6.07 \mathrm{~cm} ; \mathrm{P}<0.0001)$. There were no significant differences in operation time or the time required for SFM (Table 2).

We analyzed the subgroups by using the operation method (AR, LAR, and LAR with diverting sto$\mathrm{ma})$ to identify the oncological safety and the time required for the operation and SFM. The obtained redundancy of the colon did not differ significantly among the 3 groups. The AR operation time was shorter than that of LAR and LAR with diverting stoma (228.58 $\pm 39.69 \mathrm{~min}, 267.15 \pm 41.56 \mathrm{~min}, 294.37 \pm$ 52.17 min, respectively; $\mathrm{P}<0.0001$ ). The time required for SFM did not differ significantly among these 3 groups as well. SFM in LAR with diverting stoma required a significantly smaller portion of the entire operation time $(\mathrm{P}<0.0001)$ (Table 3$)$.

Table 2. Comparison of the data between low and high ligation of the inferior mesenteric vein

\begin{tabular}{|c|c|c|c|}
\hline & $\begin{array}{l}\text { Low ligation } \\
(\mathrm{n}=69)\end{array}$ & $\begin{array}{l}\text { High ligation } \\
(\mathrm{n}=134)\end{array}$ & P-value \\
\hline From SP $\ddagger(\mathrm{cm})$ & $24.94 \pm 6.07$ & $29.54 \pm 7.17$ & $<0.0001$ \\
\hline $\mathrm{DRM}^{\S}(\mathrm{cm})$ & $6.73 \pm 4.36$ & $6.77 \pm 4.46$ & 0.941 \\
\hline PRM" (cm) & $14.75 \pm 5.35$ & $16.91 \pm 7.27$ & 0.035 \\
\hline Specimen length $(\mathrm{cm})$ & $24.43 \pm 4.96$ & $26.53 \pm 5.74$ & 0.013 \\
\hline Operation time (minutes) & $257.06 \pm 50.65$ & $266.74 \pm 56.07$ & 0.242 \\
\hline $\begin{array}{l}\text { The time required for SFM } \\
\text { (minutes) }\end{array}$ & $26.57 \pm 11.53$ & $25.10 \pm 9.885$ & 0.175 \\
\hline $\begin{array}{l}\text { The time required for } \\
\text { SFM / Operation time (\%) }\end{array}$ & $10.39 \pm 4.40$ & $9.55 \pm 3.61$ & 0.290 \\
\hline
\end{tabular}

Table 3. Comparison of the data among the AR, LAR, and LAR with diverting stoma procedures

\begin{tabular}{|c|c|c|c|c|}
\hline & $\begin{array}{l}\text { AR } \\
(\mathrm{n}=78)\end{array}$ & $\begin{array}{l}\text { LAR } \\
(\mathrm{n}=39)\end{array}$ & $\begin{array}{l}\text { LAR with di- } \\
\text { verting stoma } \\
(\mathrm{n}=86)\end{array}$ & P-value \\
\hline From SP (cm) $^{2}$ & $26.65 \pm 7.65$ & $28.46 \pm 7.23$ & $28.57 \pm 6.92$ & 0.202 \\
\hline DRMs $(\mathrm{cm})$ & $8.75 \pm 4.04$ & $6.21 \pm 2.69$ & $5.35 \pm 4.84$ & $<0.0001$ \\
\hline PRM" (cm) & $12.29 \pm 4.23$ & $15.85 \pm 6.43$ & $19.86 \pm 6.96$ & $<0.0001$ \\
\hline $\begin{array}{l}\text { Specimen length } \\
(\mathrm{cm})\end{array}$ & $24.01 \pm 5.10$ & $26.44 \pm 5.99$ & $27.36 \pm 5.34$ & $<0.0001$ \\
\hline $\begin{array}{l}\text { Operation time } \\
\text { (minutes) }\end{array}$ & $228.58 \pm 39.69$ & $267.15 \pm 41.56$ & $294.37 \pm 52.17$ & $<0.0001$ \\
\hline $\begin{array}{l}\text { The time required } \\
\text { for SFMף (minutes) }\end{array}$ & $26.33 \pm 11.31$ & $26.38 \pm 10.46$ & $24.58 \pm 9.92$ & 0.549 \\
\hline $\begin{array}{l}\text { The time required } \\
\text { for SFM / } \\
\text { Operation time (\%) }\end{array}$ & $11.45 \pm 4.46$ & $9.86 \pm 3.18$ & $8.27 \pm 3.06$ & $<0.0001$ \\
\hline
\end{tabular}

There were no intra-operative complications during SFM such as splenic injury, bowel injury, or unexpected bleeding. In the postoperative period, 7 patients (5.6\%) who underwent LAR (39 patients) or LAR with diverting stoma (86 patients) experienced complications of the anastomosis site. Six patients $(4.8 \%)$ experienced anastomosis leakage, while one patient $(0.8 \%)$ had proximal limb ischemia. Among the 6 patients with anastomosis leakage, 5 had undergone neoadjuvant chemoradiotherapy before the surgery, LAR with a loop ileostomy. The other patient with anastomosis leakage underwent subsequent diverting loop ileostomy. The leakages in all 6 patients were minor. All of the diverting stoma of the 6 patients with anastomosis leakage were reversed ac- 
cording to our institutional protocol (approximately 3 months after creation). One patient who had proximal limb ischemia, the most serious complication noted in this study, underwent a Hartmann colostomy that has not yet been reversed.

\section{Discussion}

Tension-free and well vascularized colons are required for safe colorectal anastomosis in AR or LAR. For this reason, colonic elongation is mandatory. Two steps are involved in this process during AR or LAR: high ligation of the IMA and SFM. Although high ligation of the IMA carries the risk of compromising the blood supply to the left side of the colon, it is necessary to ligate the IMA near its origin at the level of the aorta for colonic mobilization as well as to ensure maximal lymph node clearance [9]. However, debate remains whether it is necessary to routinely or selectively mobilize the splenic flexure during AR or LAR. Some surgeons who are opposed to routine SFM question whether SFM is a mandatory step in anterior or low anterior resection [9-11]. They insist that selective SFM does not increase the risk of anastomotic leak or oncological compromise. On the other hand, other surgeons still believe that routine SFM is necessary to ensure a tension-free, well-vascularized anastomosis [12-15]. Although there are some risks associated with SFM, the frequency and magnitude of morbidity (postoperative bowel function, permanent stoma rate, and oncologic outcome) and mortality from anastomotic complications far outweigh the risks associated with SFM [14-16].

SFM is challenging because of the requirement for extensive posterior dissection while the vascular supply to the hind gut is preserved via the marginal artery. Also, SFM during laparoscopic AR or LAR may be associated with longer operation time, patient repositioning, additional port insertion, or splenic injury $[17,18]$. A mail-in survey of 35 experienced laparoscopic colorectal surgeons showed that SFM is one of the hardest procedures to perform[6]. In our study, we did not experience any complications related to SFM such as spleen injury, bowel injury, or unexpected bleeding. The mean time required for SFM was $25.61 \pm 10.56 \mathrm{~min}$, while the ratio of the time required for SFM to the entire operation time was $9.82 \% \pm 3.94 \%$. In particular, SFM in a LAR with diverting stoma $(8.27 \% \pm 3.06 \%)$ took a significantly smaller portion of the entire operation time than that in $\operatorname{AR}(11.45 \% \pm 4.46 \%)$ or LAR $(9.86 \% \pm 3.18 \%)(\mathrm{P}<$ 0.0001 ) (Table 3). In our study, all operations were performed by 3 surgeons with subspecialty for colorectal surgery in St. Vincent's Hospital of the Catholic University of Korea. In our hospital, approximately 250 operations for colorectal cancer are performed annually. We performed approximately $90 \%$ of colorectal cancer surgeries laparoscopically during this study period. These findings suggest that SFM during laparoscopic AR or LAR performed by an experienced hand is safe and feasible, rather than time-consuming as thought previously.

Until now, no report has detailed how much redundancy is actually accomplished by SFM. There were 2 recent cadaveric studies for the elongation of colon by SFM $[7,8]$. A study with 20 cadaveric models demonstrated that a mean $28.3-\mathrm{cm}$ mobilized colonic segment was achieved after full mobilization of the distal transverse colon. These authors regarded full mobilization as detachment of the distal transverse and descending colons by using the same method as ours and high ligation of the IMV [7]. Another study with 13 adult fresh cadavers demonstrated that the length of colon gained after high ligation of IMA and IMV and SFM $(28.75 \pm 5.72 \mathrm{~cm})$ altogether was greater than that after any of the other procedures (low IMA ligation with high ligation of IMV and SFM, or low IMV ligation with SFM and high IMV ligation, and etc.) [8]. However, these studies were performed with cadaveric models. In our study, the measurement of actual viable specimens in real operation field was performed. We measured the distance between the marked "A" spot and the sacral promontory after SFM in all cases. Our results were similar with those of the above-mentioned cadaveric studies. The obtained redundancy of colon by SFM from the sacral promontory, which is a control point in cadaveric studies, was $27.81 \pm 7.29 \mathrm{~cm}$.

The obtained redundancy of the colon in high IMV ligation group $(29.54 \pm 7.17 \mathrm{~cm})$ was more than that in the low IMV ligation group $(24.94 \pm 6.07 \mathrm{~cm} ; \mathrm{P}$ $<0.0001$ ). In addition, our results on the proximal and distal resection margins are sufficient for the oncological concept and complications of anastomosis site were comparable to those of earlier reports.

In our study, SFM was performed routinely even in laparoscopic AR. In laparoscopic AR, many surgeons feel that routine SFM is not necessary for the tension-free anastomosis and oncological aspects [10, $11,19]$. However, the use of SFM in laparoscopic AR may be helpful for avoiding midline skin incisions or left lower quadrant incision which require a transection of the abdominal muscles as well as for tension-free anastomosis. We harvested all of the specimens through a Pfannenstiel incision after SFM in laparoscopic AR or LAR. This allows healthy, non-traumatized tissue to lay over, potentially causing lower rates of infection. In addition, lesser tension of the incision may help prevent the development of incisional hernia. In addition, since the Pfannenstiel incision is in the lower abdomen, it is exposed to less 
tension from such activities as breathing and coughing or from increased abdominal girth, than in the upper abdomen. Tension on the midline incision is main cause of wound complications, which are likely due to vascular compromise. Consequently, the contamination of wound without appropriate blood flow leads to an even greater risk of complications. Also, the Pfannenstiel incision is more cosmetic than a midline or left lower quadrant transverse incision [20]. In this study, there were 2 wound infections $(0.9 \%)$, one wound hematoma $(0.5 \%)$, and no incisional hernias. Despite the lack of an objective tool for comparing wound pain, we felt that patients with the Pfannenstiel incision were more comfortable than those without. Although further study is required to identify the advantage of the Pfannenstiel incision, our experience suggests that this incision following SFM during laparoscopic AR or LAR may be a better surgical option.

This study has the limitation of selection bias due to the fact that only patients with availiable data of colon measurement were included not the all patients who underwent laparoscopic AR and LAR. However, it also showed the objective lengths of the viable specimens gained by colonic mobilization with SFM in laparoscopic AR or LAR.

SFM during LA or LAR resection is a safe and feasible option. We think that we can gain about 27 $\mathrm{cm}$ redundancy of the colon by SFM. If high IMV ligation is performed, one can gain approximately $5 \mathrm{~cm}$ more than low IMV ligation performed. SFM and high IMV ligation provided an approximately $30 \mathrm{~cm}$ redundancy of the colon. Therefore SFM can give a safe anastomosis following by proper oncological resection for the treatment of sigmoid and rectal cancer. Also, these results may be worthy as a reference value for selective SFM.

\section{Acknowledgement}

We appreciate Seung Hyun Cho who helped data collection and Sung Min Kim who illustrated schematic figure.

\section{Competing Interests}

The authors have declared that no competing interest exists.

\section{References}

1. Kulaylat MN, Dayon MT. Surgical Complications. In: Townsend CMJr, Beauchamp RD, Evers BM, Mattox KL, eds. Sabiston Textbook of Surgery, 19th eds. Philadelphia: Saunders, an imprint of Elsevier Inc. 2012:315-318

2. Konish T, Watanabe T, Kishimoto J, Nagawa H. Risk factors for anastomotic leakage after surgery for colorectal cancer: results of prospective surveillance. J Am Coll Surg. 2006;202:439-444

3. Veldkamp R, Kuhry E, Hop WC, et al. Laparoscopic surgery versus open surgery for colon cancer: Short-term outcomes of a randomised trial. Lancet Oncol 2005;6:477-484

4. Katory M, Tang CL, Koh WL, et al. A 6-year review of surgical morbidity and oncologic outcome after high anterior resection for colorectal malignancy without splenic flexure mobilisation. Colorectal Dis 2008;10:165-169

5. Benseler V, Hornung M, Iesalnieks I, et al. Different approaches for complete mobilization of the splenic flexure during laparoscopic rectal cancer resection. Int J Colorectal Dis. 2012;27:1521-1529

6. Jamali FR, Soweid AM, Dimassi H, et al. Evaluating the degree of difficulty of laparoscopic colorectal surgery. Arch Surg. 2008;143:762-767

7. Araujo SE, Seid VE, Kim NJ, et al. Assessing the extent of colon lengthening due to splenic flexure mobilization techniques: a cadaver study. Arq Gastroenterol. 2012;49:219-222

8. Thum-Umnuaysuk S, Boonyapibal A, Geng YY, Pattana-Arun J. Lengthening of the colon for low rectal anastomosis in a cadaveric study: how much can we gain? Tech Coloproctol. 2012; DOI: 10.1007/s10151-012-0930-6

9. Chand M, Miskovic D, Parvaiz AC. Is splenic flexure mobilization necessary in laparoscopic anterior resection? Dis Colon Rectum. 2012;55:1195-1197

10. Park JS, Kang SB, Kim DW, et al. Laparoscopic versus open resection without splenic flexure mobilization for the treatment of rectum and sigmoid cancer: a study from a single institution that selectively used splenic flexure mobilization. Surg Laparosc Endosc Percutan Tech. 2009;19:62-68

11. Marsden MR, Conti JA, Zeidan S, et al. The selective use of splenic flexure mobilization is safe in both laparoscopic and open anterior resections. Colorectal Dis. 2012;14:1255-1261

12. Dixon AR, Maxwell WA, Holmes JT. Carcinoma of the rectum: a 10-year experience. Br J Surg 1991;78:308-311

13. Finan PJ. Splenic flexure mobilisation for anterior resection performed for sigmoid and rectal cancer: why I (nearly) always mobilize the splenic flexure in rectal cancer surgery. Ann R Coll Surg Engl 2008;90:638-42.

14. Ludwig KA, Kosinski L. Is splenic flexure mobilization necessary in laparoscopic anterior resection? Another view. Dis Colon Rectum. 2012;55:1198-1200

15. Hiranyakas A, Da Silva G, Denoya P, et al. Colorectal anastomotic stricture: Is it associated with inadequate colonic mobilization? Tech Coloproctol. 2012;[Epub ahead of print]

16. Taflampas P, Christodoulakis M, Tsiftsis DD. Anastomotic leakage after low anterior resection for rectal cancer: facts, obscurity, and fiction. Surg Today. 2009;39:183-818

17. Gezen C, Altuntas YE, Kement M, et al. Complete versus partial mobilization of splenic flexure during laparoscopic low anterior resection for rectal tumors: a comparative study. J Laparoendosc Adv Surg Tech A. 2012;22:392-396

18. Akiyoshi $\mathrm{T}$, Kuroyanagi $\mathrm{H}$, Oya M, et al. Factors affecting difficulty of laparoscopic surgery for left-sided colon cancer. Surg Endosc. 2010;24:2749-2754

19. Brennan DJ, Moynagh M, Brannigan AE, et al. Routine mobilization of the splenic flexure is not necessary during anterior resection for rectal cancer. Dis Colon Rectum. 2007;50:302-307

20. Orcutt ST, Balentine CJ, Marshall CL, et al. Use of a Pfannenstiel incision in minimally invasive colorectal cancer surgery is associated with a lower risk of wound complications. Tech Coloproctol. 2012;16:127-132 